\title{
Desarrollo de la competencia argumentativa en foros de discusión en línea: una propuesta constructivista
}

\author{
Yunuen I. Guzmán-Cedillo*, Rosa-del-Carmen Flores-Macías y Felipe Tirado-Segura
}

Universidad Nacional Autónoma de México Facultad de Psicología

\begin{abstract}
Resumen: En este escrito se plantea la necesidad de revisión, definición y caracterización de la competencia argumentativa desde el enfoque constructivista, así como el planteamiento de su desarrollo en foros de discusión en línea, por considerarlos espacios donde se promueve el debate con la finalidad de llegar a acuerdos. A partir de la revisión de documentos sobre competencias, argumentación, pensamiento crítico y foros de discusión se propone a la competencia argumentativa como la articulación de doce elementos agrupados en los tres componentes (conocimientos, habilidades y actitudes) desplegados en argumentos dialógicos que derivan en un desarrollo de pensamiento crítico producto de un compromiso cognitivo individual y con los participantes, en el que la temática y el diseño educativo son fundamentales.

Palabras clave: Competencias; argumentación; foros de discusión en línea; enfoque constructivista; educación por competencias.
\end{abstract}

\begin{abstract}
Title: Argumentative competences development in online discussions forums.

Abstract: Setting out an analysis and definition of argumentative competence from a constructive perspective is the objective in this paper. Furthermore it proposes online forums which promote debate as an appropriate environment to develop this kind of competences. Argumentative competence concept suggested here comes from an analysis of different documents and researches about critical thinking, argumentation, competences and online forums. This concept involves at list twelve elements to group into three competences components (knowledge, skills and attitudes) to deploy in dialogic arguments that develops a critical thinking in an individual and group cognitive engagement where the topic and instructional design are fundamental.
\end{abstract}

Key words: Competences; argumentation; online discussions; constructivism; skills.

\section{Introducción}

Actualmente, las instituciones de educación superior tienen como encomienda lograr que los estudiantes dominen los conocimientos propios de su profesión; tengan una concepción compleja de los problemas y desarrollen un pensamiento científico para aproximarse a su análisis y solución; posean habilidades para resolver problemas; desarrollen una capacidad de trabajo en colaboración; orienten éticamente su ejercicio profesional; y, además, den continuidad a su propia formación con el objetivo de mejorar su labor. En su conjunto a toda esta integración, asimilación y uso de recursos adquiridos en la formación profesional se le ha llamado competencia.

En este panorama, las Tecnologías de la Información y de la Comunicación (TIC) ${ }^{1}$ juegan un papel clave, ofreciendo nuevos contextos y posibilidades para el desarrollo de dichas competencias. Las nuevas tecnologías generan espacios de comunicación idóneos para la formación de profesionales dentro de modalidades educativas completamente a distancia o híbridas.

La incorporación de las nuevas tecnologías ha puesto de manifiesto la necesidad de que los estudiantes aprendan a comunicarse mediante distintas herramientas. Específicamente, la argumentación en la formación universitaria en línea ha sido objeto de estudio por sus implicaciones para comunicar ideas, adquirir conocimiento, desarrollar un pensamiento científico y crítico, además de generar conocimiento para enfrentar problemas complejos.

* Dirección para correspondencia [Correspondence address]: Yunuen Ixchel Guzmán-Cedillo. Universidad Nacional Autónoma de México Facultad de Psicología. Av. Universidad núm. 3004, Col. Copilco-Universidad, C.P. 04510, Delegación Coyoacán, México DF. (México). E-mail: yunuenixchel@hotmail.com
Dada su relevancia, el presente trabajo se centra en el estudio de la competencia argumentativa planteando para su observación y estudio el foro de discusión en línea como un escenario específico de desarrollo.

Una competencia pasa por diferentes niveles de desempeño en situaciones que requieren del despliegue de sus componentes (Amestoy, 2002; Perrenoud, 2009; Vergnaud, 2006, Tardif, 2006). Este desarrollo en el desempeño se da gracias a las oportunidades de práctica y reflexión que se brindan para movilizar los recursos y lograr una acción eficaz para responder a situaciones que se valoran como problema en un contexto particular (Jonnaert, 2000; Jonnaert, Barrette, Masciotra y Yaya 2008; Perrenoud, 2004, 2008, 2009; Coll, 2009).

Desde una perspectiva psicológica y lingüística, se sabe que la habilidad para argumentar tiene un desarrollo en situaciones donde se da la posibilidad de un argumento dialógico (Kuhn, 1992, 2000; Kuhn y Lao, 1998; Kuhn y Park, 2005; Willard, 1983; Van Eemeren y Grootendorst, 1992; Peon, 2004). En este sentido la literatura reporta que un escenario donde se facilita este tipo de argumento es el foro de discusión en línea (Kuhn, Goh, Iordanou y Shaenfiel, 2008; Mason y Scirica. 2006; Ying-Hua, 2006; Fu-Ren, Lu-Shih y Fu-Tai, 2009; Hammond, 2005; Kanuka, Rourke y Laflamme, 2007), de este espacio se han realizado una serie de investigaciones sobre los patrones de interacción, los tipos y niveles de discusión, la forma de participación y cómo se integra una comunidad (Gútierrez, 2004; Häkkinen y Järvela, 2004; Veerman, 2000; Garrison y Anderson, 2005); estos estudios reportan que la forma de argumentar de los participantes se modifica en los foros de discusión. Para comprender cómo ocurre y de qué forma se manifiesta dicha evolución, el presente trabajo propone una aproximación a la ar-

2 Entendidas como aquellos artefactos tecnológicos que hacen posible el almacenamiento, e interpretación y distribución de información. 
gumentación diferente a las que se han planteado, al conceptuarla como una competencia desde el enfoque constructivista.

Bajo este enfoque, la competencia supone que son varios los recursos que el individuo pone en marcha en una acción situada al elaborar un argumento. La concepción de competencia argumentativa obedece a una integración de conocimientos, habilidades y actitudes al interactuar en una argumentación dialógica presente en un foro de discusión cuyo objetivo es promover la deliberación alrededor de un tema.

Al tener al foro de discusión como escenario, la competencia argumentativa se entiende como la integración de conocimientos, habilidades y actitudes, movilizados en la defensa o rechazo de una aseveración, hecho o conclusión a través de una exposición coherente, fundamentada y sistemática con el objeto de plantear una postura a la vez que se comparten significados y se negocia un consenso.

La exposición de este planteamiento se organiza en cinco apartados. El primer apartado esboza una panorámica general del enfoque de competencias en educación al comentar grosso modo el traslado del concepto del mundo laboral al educativo. El segundo apartado da una explicación de lo que es la competencia argumentativa, ya que analiza y comenta estudios realizados bajo dos perspectivas básicas sobre argumentación: la lingüística y la psicológica. En el tercer aparta- do se presenta una revisión de investigaciones sobre foros de discusión en línea como promotores de la argumentación. El cuarto y más sustancial se centra en dar una serie de elementos que fundamenta la constitución de la definición y desarrollo de la competencia argumentativa en los foros de discusión en línea. El último apartado ofrece una serie de conclusiones acerca de este tópico.

A partir de lo anterior, este artículo busca caracterizar la competencia argumentativa desde el enfoque constructivista y plantear como situación de desarrollo a los foros de discusión en línea.

\section{El enfoque por competencias en educación}

El enfoque por competencias en investigación educativa se ha extendido en virtud de las expectativas de mejora en la eficacia y la calidad de los procesos de enseñanzaaprendizaje, lo cuales han sido base de una serie de reformas en los diferentes niveles educativos en distintos países.

Son tres los enfoques que se reconocen como parte del estudio de las competencias en el desempeño eficiente: el conductista, el genérico y el cognitivo (Mulder y Collings, 2007; Mulder, Weigel y Collings, 2008; Delamare y Wingerton, 2005); en la Tabla 1 se describen las características principales de cada uno.

Tabla 1. Enfoques sobre las competencias.

\begin{tabular}{|c|c|c|c|}
\hline & Conductista & Genérico & Cognitivo \\
\hline Objetivo & $\begin{array}{l}\text { Definir las características desde una re- } \\
\text { visión funcional del desempeño eficien- } \\
\text { te. }\end{array}$ & $\begin{array}{l}\text { Identificar las características principales } \\
\text { en términos de habilidades necesarias en } \\
\text { el desempeño eficiente. }\end{array}$ & $\begin{array}{l}\text { Identificar los recursos mentales para } \\
\text { un buen desempeño. }\end{array}$ \\
\hline Contexto & Trabajo & Contextos dinámicos (laboral o escolar) & Situaciones problema \\
\hline Perspectivas & Múltiples escuelas derivadas & Holística & $\begin{array}{l}\text { Inteligencia, procesamiento de la in- } \\
\text { formación, desarrollo, y socioconstruc- } \\
\text { tivismo }\end{array}$ \\
\hline
\end{tabular}

Son distintos los objetivos y contextos en los que se dan las competencias según el enfoque que se adopte así como las diferentes vertientes de estudio elaboradas a partir de una visión particular de aprendizaje y desarrollo de la competencia.

No obstante sus diferencias, Weinert (2001) comenta que los enfoques coinciden en cuatro aspectos: 1) la movilización de recursos, 2) su articulación e interrelación, 3) la naturaleza contextual de los procesos de adquisición y aplicación, además de 4) la importancia de la actuación eficaz.

El concepto de competencia nace en la empresa en 1982 con McClelland (En Mulder, Weigel y Collins, 2008), en los años 90 es retomado por las universidades e institutos para justificar la preparación a los ámbitos laborales. En 1996 el Informe Delors abandera la noción de competencia al reivindicar y replantear la función de la educación para formar a los individuos de la sociedad de la información. Propone como objetivos primordiales, los llamados cuatro pilares de la educación: aprender a conocer, es decir, adquirir los instrumentos de la comprensión; aprender a hacer, para poder influir sobre el propio entorno; aprender a ser, un proceso fundamental en términos de disposición aunada a valores éticos; y aprender a vivir juntos, para participar y cooperar con los demás en todas las actividades humanas que promueven el desarrollo en diferentes áreas.

Después del informe se llevan a cabo varios pronunciamientos en relación con el desarrollo de estos pilares (Delgado, Borge, García, Oliver y Salomon, 2005), iniciando con la Declaración de la Sorbona (1998) cuyos acuerdos son base de la Declaración de Bolonia (1999) que abandera un nuevo enfoque de titulación en la educación superior basado en competencias, el cual sirve a su vez en la construcción del proyecto Tuning (2000), el cual toma una perspectiva genérica de competencias para lograr el objetivo de armonizar la educación superior europea. Después (2001) se constituye el Espacio Europeo de Educación Superior (EEES), con el fin de revisar los logros que han tenido en cuanto a la ambiciosa labor de llegar a acuerdos en la formación profesional de las Instituciones Europeas (Coll, 2007).

Posteriormente, mediante el proyecto DeseCo (Desarrollo de competencias) en 2002 y en 2003 con el Programa pa- 
ra la Evaluación Internacional de Estudiantes (PISA), la Organización de Cooperación y Desarrollo Económico (OCDE) propone identificar, describir y evaluar las competencias que debiesen sustentar los aprendizaje en la educación básica (En Delgado y cols., 2005).

En estos momentos el enfoque educativo por competencias, enmarca las diferentes políticas de calidad para la educación, orientando las reflexiones sobre el proceso educativo y sus finalidades (Tobón, 2006). Frente a esto, el enfoque constructivista de competencias estipula una educación flexible, centrada en el aprendiz, quien es considerado un ser activo que desarrolla capacidades articuladas con conocimientos, habilidades y actitudes; las cuales exigen más protagonismo y compromiso del estudiante (González, Wageenar y Beneitone, 2004).

Con una visión integral del uso de recursos como habilidades, conocimientos y actitudes que se reflejen en el desempeño eficiente del estudiante en actividades de la vida cotidiana vinculadas al aula, este enfoque aporta al paradigma educativo actual, flexibilidad y riqueza (Coll, 2009).

\section{La competencia argumentativa}

\section{La perspectiva lingüística}

$\mathrm{Al}$ definir de manera puntual a la competencia argumentativa, Weigand (2006) y De Zubiría (2006) recuperan la visión pragmadialéctica (Van-Emeren y Grootendorst,1992; Van Eemeren y Houtlosser, 2007; Van Eemeren, Houtlosser y Snoeck, 2008) al explicarla como capacidad de organización de una serie de elementos como son: la evaluación de la información, la resolución de contradicciones, la reconsideración de afirmaciones con base en aspectos sociales, puestos a trabajar en una situación particular además del convencimiento sustentado en evidencia que se da en un argumento. Bajo este enfoque se ha destacado la necesidad de revisión de los procedimientos y las actitudes que derivan en una dimensión ética (Posada, 1999).

En la perspectiva lingüística la evidencia de la argumentación vista como competencia se constituye en propuestas de modelos teóricos o disertaciones de las implicaciones que tendría en la construcción del discurso argumentativo (Weigand, 2006). La evidencia empírica se recarga en estudios donde se analiza la habilidad discursiva para la construcción con base en evidencias de un discurso coherente con el objetivo comunicativo de disuadir y convencer al interlocutor de adherirse a una postura frente a una temática, hecho u opinión (acento en el sentido retórico del argumento) (Peon, 2004; Willard, 1983; Sampson y Clarck; 2008).

Desde esta perspectiva, se han desarrollado distintos modelos (nueva retórica de Perelman y Olbrech-Tyteca, pragma-dialéctica de Van-Eemeren, escuela constructivista de Grize y retórica racional de Toulmin) para evaluar las vías en que un individuo genera un argumento. Es frecuente que esos modelos se refieran a tres aspectos fundamentales: 1. La estructura o complejidad del argumento (componentes), 2.
El contenido del argumento (adecuación de los componentes) y 3. Naturaleza de la justificación (validación de las aseveraciones) (En Sampson y Clarck, 2008).

Modelos que subrayan una visión donde la calidad del argumento es evaluada gracias a las habilidades verbales manifestadas.

En consecuencia, las investigaciones con perspectiva lingüística sobre la argumentación han hecho aportaciones sobre el diseño y evaluación de la efectividad de situaciones de aprendizaje que promueven las habilidades discursivas para construir un argumento; el análisis de los elementos que constituyen el proceso argumentativo (uso de evidencias, contraargumentación y refutación) y la comparación del nivel de discusión así como la calidad de los argumentos construidos.

No obstante la relevancia de estas aportaciones en el campo de la argumentación la perspectiva lingüística enfatiza el procedimiento para construir un argumento pero deja de lado la articulación de conocimientos y actitudes involucrados en la elaboración reflexiva del argumento; articulación necesaria para ver la capacidad de argumentar como competencia.

\section{Perspectiva psicológica}

A diferencia de la perspectiva lingüística, argumentar se ha revisado en psicología como una facultad individual cuyo desarrollo requiere de un aprendizaje sistemático y deliberado para defender una postura de manera razonada ya sea en un argumento, contraargumento o refutación ${ }^{2}$, donde el proceso central es la reflexión del propio pensamiento al sostener razonadamente una idea, sobre todo en diálogos sociales (argumentos dialógicos) considerados como caminos para externalizar el pensamiento y clarificar un punto de vista donde los otros fungen como espejos (Kuhn, 1992).

Con respecto a la investigación psicológica sobre pensamiento argumentativo donde Kuhn es pionera, son varios los trabajos desarrollados alrededor de ambientes diseñados con el objeto de identificar los elementos que la promueven.

En una de sus investigaciones, Kuhn (1992) describe el proceso de razonamiento al construir un argumento subrayando las creencias, opiniones y juicios que las personas adoptan con la exposición de su punto de vista sobre diferentes temas de importancia social, descubre que ese proceso no depende del género o la edad, pero el nivel educativo posee cierta influencia.

En otro estudio, Kuhn y Lao (1998) ubican que la polarización promueve la modificación de creencias base de un argumento en una dirección más compleja, porque la nueva evidencia es un mecanismo de cambio conceptual.

Bajo esta línea de revisión del pensamiento argumentativo, Kuhn, Cheney y Weinstock (2000) construyen un mode-

${ }^{2} \mathrm{El}$ argumento es el producto de una elaboración argumentativa, el contraargumento es la respuesta que se da al argumento que manifiesta el interlocutor y la refutación se refiere a la interpelación del contraargumento (Nussbaum, Sinatra y Poliquin, 2008). 
lo de desarrollo cognitivo. El modelo utilizado ubica tres tipos de pensamiento de los individuos al construir argumentos en interacción con otros: el absolutista que ve al conocimiento como una entidad objetiva, localizado en el mundo externo que puede ser conocido con certeza; el multiplicista que ve al conocimiento como parte del entendimiento subjetivo lo que proporciona un grado de incertidumbre donde todas las opiniones pueden ser correctas; el evaluativista que reintegra la dimensión objetiva del conocimiento, pues para enfrentar la incertidumbre se deben emitir juicios para legitimar posiciones al argumentar con base en evidencias.

En esta misma línea de entendimiento de los argumentos como manifestaciones de desarrollo cognitivo, Kuhn y Park (2005) proponen un modelo que contiene cuatro posiciones de pensamiento. En la primera (realista) las aseveraciones son copias de la realidad externa (se repite lo observado y escuchado), el conocimiento proviene de un recurso externo, cierto e incuestionable, las siguientes etapas son la absolutista, multiplicista y evaluativista (ya explicadas). El modelo involucra elementos cognoscitivos en el proceso de argumentar al valorar en la aseveración el origen del conocimiento y el pensamiento crítico.

En sintonía con los anteriores estudios de desarrollo, Mason y Sirica (2006), encontraron que los tres componentes de la argumentación (producir argumentos, contraargumentos y refutaciones) correlacionaron positivamente con el desarrollo cognitivo (entendimiento epistemológico), observaron que el interés no se relaciona con ellos y el conocimiento previo no emergió de manera clara en el estudio.

En otra investigación, Nussbaum, Sinatra y Poliquin (2008) examinaron el efecto del desarrollo cognitivo (etapas) y la exposición a los criterios de un argumento científico, para determinar si la naturaleza y calidad de los argumentos se incrementaba. Sus resultados apoyan que el desarrollo cognitivo (evaluativista, multiplicista y absolutista) determina la capacidad de considerar la evidencia, los puntos de vista alternativos, emitir juicios y resolver inconsistencias.

En otro estudio, Kuhn, Iordanou, Pease y Wirkala (2008) mencionan que un elemento fundamental para desarrollar habilidades de pensamiento científico es la habilidad de realizar una argumentación debido a que la reflexión al construir un argumento coexiste con las habilidades mostradas al describir un procedimiento utilizado en ciencias.

En el caso de la psicología, argumentar está ligado al desarrollo cognitivo y ha sido revisado como una habilidad que se desarrolla en situaciones educativas diseñadas exprofeso para ser capaz de defender una postura de manera razonada en un argumento, contraargumento o refutación.

\section{El papel de los foros de discusión en línea en el estudio de la argumentación}

El desarrollo de las TIC brinda nuevos contextos y posibilidades para el desarrollo de la competencia argumentativa, escenarios que posibilitan investigar de manera integral tanto los recursos psicológicos como los comunicativos además del diseño instruccional implicados en el desarrollo de la competencia argumentativa como objeto de estudio (Clarck, Stegmann, Weinberger, Muhsin y Erkens, 2008; Rodríguez, 2004; Lewis, 2005).

Al respecto, los foros de discusión en línea se utilizan de diferente manera y número en programas de formación universitaria. Han servido para propiciar la interacción social durante el aprendizaje; para la discusión de tareas o trabajos de evaluación; para negociar la toma de decisiones en un equipo de trabajo o para desarrollar una estrategia educativa (Mazzolini y Maddison, 2003); también han sido propuestos para propiciar el debate de ideas y posturas en torno a un tema dado (Veerman, Andriessen y Kanselaar, 2000; Coffin y Hewings, 2005).

Los foros han mostrado ser un escenario idóneo al permitir indagar a profundidad la forma y cambios en la generación de argumentos (Lewis, 2005; Fu-Ren et al., 2009). Uno de los puntos positivos del foro es que permite el registro, organización y etiquetación de las aportaciones de los participantes, con lo que los miembros saben de qué trata el comentario y quién lo realizó (Lewis, 2005; Fu-Ren, Lu-Shen y Fu-Tai, 2009). Otro aspecto positivo reportado es su carácter asincrónico, dado que el foro de discusión da la oportunidad de pensar y repensar las ideas sin tener la presión de participación inmediata que implica el debate sincrónico, además su historia es accesible en cualquier momento. El ser asincrónico es una característica fuerte y potencial del foro.

Los estudios sobre argumentación en foros de discusión resaltan que la construcción de un argumento es un proceso que implica una serie de habilidades de razonamiento y verbales como parte de un pensamiento de orden superior a partir del cual se analizan, critican o sopesan puntos de vista frente a un tema particular. Argumentar de manera asincrónica en el foro de discusión favorece el examen riguroso de las cualidades y circunstancias de un planteamiento, ya que en la discusión se dan razones para comparar posiciones, determinar relaciones, realizar juicios y tomar decisiones (Guzmán-Cedillo, 2010).

En el mismo sentido, Coffin (2004) comenta que los estudiantes analizan la retroalimentación a sus contribuciones y cómo participarán de manera más cuidadosa que en las discusiones presenciales, pues se dan tiempo de pensar antes de dar la réplica, igualmente permite al profesor la intervención con objeto de ampliar la perspectiva de los estudiantes. La comunicación con base textual y asíncrona es benéfica para mantener en curso y centrada una discusión (Marttunen, 1997, 1997; Lewis, 2005). Por tanto, los foros son oportunidades de reflexión (Coffin y Hewings, 2005).

Asimismo, el foro de discusión abre la oportunidad de una participación equitativa (efecto de participación igualitaria), que repercute en la actitud hacia la participación en un debate, debido en gran parte a que los miembros del grupo pueden dar una respuesta en cualquier momento (Joiner y Jones , 2003).

Diferentes investigaciones se han abocado a comprender las características del foro en términos de diseño en las 
características de argumentación de los alumnos en foros de discusión, sus hallazgos son aportaciones importantes para dar sustento a la oportunidad que brindan los foros para el desarrollo de la competencia argumentativa.

En el caso de Coffin, North y Martin (2009) encontraron que el cuestionamiento es vital al elaborar una contraargumentación. En su estudio reportan que, además de ser escasa, se presentó cuando se había solicitado abiertamente se explicará la afirmación. Además encontraron que el manejo y tipo de evidencia para defender una posición y la existencia de un compromiso para revisar diferentes puntos de vista fueron resultado de foros de discusión diseñados ex profeso como espacios para el ensayo y desarrollo de habilidades argumentativas acompañados de la oportunidad brindada a los estudiantes de revisar la estructura de un buen argumento, previo a la participación en el foro.

Kuhn, Goh, Iordanou y Shaenfiel (2008) apoyan el punto de que el compromiso social en una argumentación dialógica es un elemento que se reconoce como valioso en la construcción de conocimiento. Mientras que Kuhn, Iordano, Pease y Wirkala (2008) comentan que el ambiente mediado por la computadora permite la reflexión. En sus resultados Golanics (2008) sugiere que el intercambio con los compañeros y la meta clara a partir de las instrucciones, definen el nivel de compromiso asumido por los participantes al profundizar la discusión.

En términos de pensamiento argumentativo Kuhn y Udell (2003) mencionan que tanto el dar oportunidades de ejercitar la construcción de argumentos mediante debate, como el que los estudiantes conozcan las metas argumentativas así como las características de un buen argumento, incrementan su rendimiento en cuanto a la calidad y cantidad de razones dadas en la construcción de un argumento.

A la luz de los resultados de investigaciones sobre foros de discusión en línea (Coffin y Hewings, 2005; Coffin, 2004; Joiner y Jones, 2003; Hammond, 2000, Hammond y Wiriyapinit, 2005; De Smet, 2009; Ying, 2006), se enmarcan una serie de fortalezas que posee el foro: provee de una comunicación que permite la reflexión (tanto propia y de otros), reduce la ansiedad del status al participar, tiene potencial de generar aprendizaje colectivo, promueve la construcción del conocimiento, los participantes cuidan las evidencias utilizadas, promueven habilidades argumentativas, promueve el pensamiento crítico al permitir un debate, brinda un sentido de comunidad, incrementa la autonomía de pensamiento y posibilita el compromiso cognitivo del estudiante.

En resumen, el foro de discusión es un espacio que permite la generación de razonamientos alrededor de afirmaciones (Veerman, Andriessen y Kanselaar, 2000; Lewis, 2005). Es un escenario donde se pueden revisar posiciones frente a un hecho o situación con respecto a otras, gracias al proceso de involucramiento en una deliberación para resolver diferencias de opinión así como productos simbólicos construidos o criticados durante un cuestionamiento (Nussbaum, Sinatra y Poliquin, 2008).
En general los estudios anteriores avalan la necesidad de comprender las participaciones de los estudiantes en foros de discusión considerando como foco de análisis el diseño educativo y la calidad de los argumentos. Estos estudios no han contemplado la comprensión de la argumentación como una competencia donde no sólo se manifiesten habilidades argumentativas sino conocimientos del tema y actitudes al interactuar en el foro de discusión articuladas al momento de participar en un argumento dialógico. Si bien estas investigaciones han hecho contribuciones importantes para dar más claridad en el campo, aún no se ha planteado la posibilidad de valorar y explicar el desarrollo de la argumentación como competencia que brinda el foro de discusión en línea.

\section{La competencia argumentativa en foros de discusión en línea: el desarrollo de una acción experta}

Hasta aquí tanto en el campo de competencias, argumentación y foros de discusión en línea se ha observado una escasez de estudios que contemplen la competencia argumentativa de manera específica. Sin embargo, con base en esta revisión, se han identificado al menos doce recursos vinculados estrechamente en la construcción de un argumento, articulados de tal forma que manifiesten una competencia en ejecución (véase Figura 1).

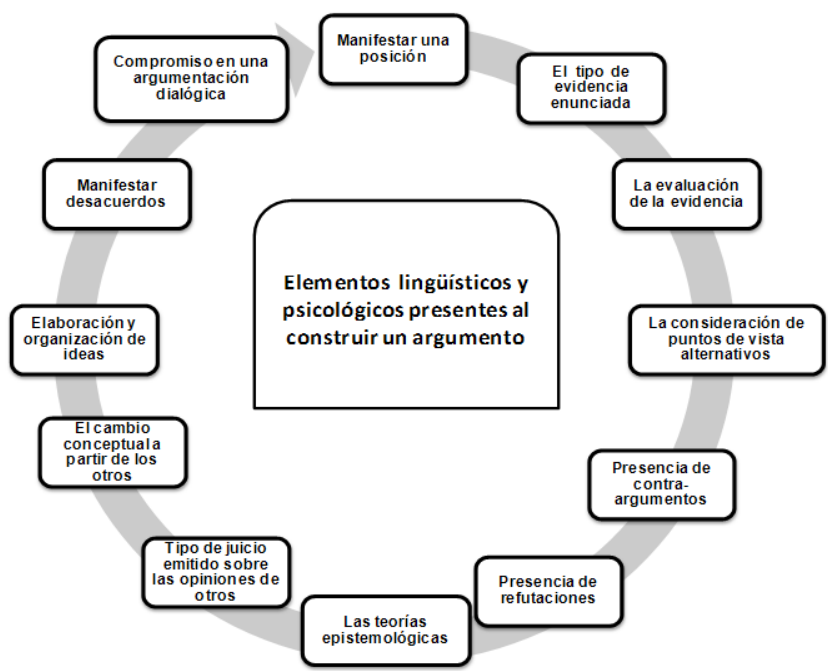

Figura 1. Recursos presentes en la competencia argumentativa.

Para explicar esta articulación de recursos al elaborar un argumento, se propone el enfoque constructivista de competencias, el cual provee de un marco para comprender el desarrollo de la argumentación como una acción experta que es posible promover en foros de discusión en línea diseñados ex profeso. Esta explicación se vierte en los siguientes apartados. 


\section{Enfoque constructivista}

El marco constructivista derivado directamente del enfoque cognitivo de competencias es el que se propone para dar cuenta del desarrollo de la competencia argumentativa. Desde este enfoque, la competencia se define como una facultad de movilizar un conjunto de recursos cognoscitivos para enfrentar con pertinencia y eficacia a una familia de situaciones (Perrenoud, 2004).

El enfoque constructivista de competencias permite entender la construcción de un argumento en situación dialógica, como se da en el foro de discusión, cuando de manera integrada y articulada todos los elementos mencionados en la Figura 1 se constituyen en una acción experta que lleva a cabo un individuo al exponer de manera coherente, fundamentada y sistemática una aseveración, hecho o conclusión para plantear una postura, a la vez que muestra una actitud frente a la temática y al interlocutor en una situación donde se intercambian y comparten significados gracias a la oportunidad de debatir y llegar a acuerdos.

Las principales características de la competencia desde la visión constructivista son la puesta en marcha de una capacidad, el desarrollo (aprendizaje) de esa capacidad hasta lograr un desempeño eficiente y el contexto en el que se despliega y lo dinámica que es la capacidad al poder orquestar los recursos cognitivos en una acción.

\section{Desarrollo de la competencia: una acción experta}

El desarrollo de una competencia implica un proceso de evolución de la pericia en términos de conceptualizar y representar lo que se hace en muchas oportunidades de ejercicio, donde está implicado un saber personal que se elabora en la acción misma del sujeto quien razona las condiciones en las que se encuentra, evalúa los obstáculos y elige la mejor solución.

La competencia se muestra cuando se ha logrado por parte del sujeto una adaptación a la situación la cual depende de circunstancias relativas que el individuo se vuelve capaz de evaluar. En ese momento el sujeto puede hacer algo para lo cual no era capaz, lo que se vuelve la evidencia de un nivel de desempeño competente (Vergnaud, 2006).

En su desarrollo, una competencia pasa por razonamientos explícitos, decisiones conscientes, dudas, ensayos y errores, por lo que cuando se observa una automatización total y se alcanza un nivel de eficiencia superior se trata más bien de una forma compleja del esquema que se ha estabilizado. Un experto es competente porque domina rápido y con seguridad las situaciones normales, ya que dispone de esquemas complejos que entran de manera automática e inmediata en acción. Además, el experto es capaz de un esfuerzo de reflexión razonable para coordinar y distinguir velozmente los esquemas pertinentes para enfrentar situaciones nuevas: “mientras más experto se es, menos se razona y más se activan los conocimientos adecuados y funcionalmente estructurados..." (Perrenoud, 2008, p. 33).
Bordieu (En Shön, 1998) lo explica a partir de una acción automatizada o hábito (babitus) que permite enfrentar variaciones menores de manera eficaz mediante una adaptación integrada a la acción, a través de un ajuste del esquema a la singularidad de la situación, cuando ésta es muy distinta a lo que es manejable por medio de una adaptación simple de esquemas; es decir, se da una toma de consciencia del obstáculo y de los límites de los recursos disponibles, de ahí que se inicie un proceso de búsqueda que en una situación exitosa da lugar a una acción original. Así es como se explica la capacidad de movilización de recursos para dar respuestas originales y eficaces a nuevos problemas o tareas encomendadas no sólo en el foro de discusión sino en innumerables situaciones.

En este proceso de adaptación a diferentes circunstancias, no hay un saber movilizar universal, por lo que en una competencia, una función por realizar es la asociación de los recursos movibles para lograr una acción eficaz; en efecto, un recurso no es exclusivo de una competencia, pues a su vez puede funcionar como recurso de otra y así es como se puede hablar de competencias y subcompetencias en una acción donde se articulan conocimientos, habilidades y actitudes. Es decir, los recursos revisados en la Figura 1 no sólo son utilizados en la competencia argumentativa sino en otras circunstancias y situaciones diversas pero que se pueden enfrentar por una extrapolación de esquemas, construidos en los foros de discusión que evalúan la situación y pertinencia de la acción por realizar.

\section{La competencia argumentativa en foros de dis- cusión en línea}

Al trasladar estos razonamientos sobre el desarrollo de la competencia al foro de discusión en línea, este proceso ocurriría cuando el participante analiza sus aportaciones y pensamientos a la luz de las aportaciones de otros, sopesa y reflexiona cómo será su siguiente participación anticipando el efecto que tendrá, todo ello acompañado de un manejo de conocimientos sobre lo que se discute y una actitud que le permite atender y entender las preocupaciones de los compañeros frente a la temática alrededor de la cual se ha establecido una deliberación.

Para movilizar los recursos ya enunciados (véase Figura 1), esta acción experta demanda una serie de operaciones mentales de alto nivel como son esquemas de conocimiento, percepción, evaluación, operación, todos ellos parte de una estructura cognitiva compleja que permite la abstracción, la relación, la comparación y el razonamiento, los cuales dan pie a la reflexión que permite la toma de decisiones en una situación evaluada como compleja (Perrenoud, 2008). Es decir, la competencia argumentativa involucra más elementos que la habilidad argumentativa (cuya característica principal es una destreza automatizada), la cual sería sólo uno de los recursos movilizados en la competencia argumentativa.

La competencia se adapta a diversas circunstancias de tal forma que al ser competente cuando se argumenta en los fo- 
ros de discusión, el individuo puede hacerlo en situaciones familiares o novedosas, como es la apertura de nuevos temas de debate o tareas distintas por realizar en el foro que exijan el despliegue de los recursos que componen su competencia argumentativa.

$\mathrm{Al}$ entender la forma en que se constituye una competencia se puede comprender cómo los doce recursos se articulan para constituir la acción experta manifestada en la competencia argumentativa. En la Figura 2 se muestran los doce componentes que a la luz de las investigaciones revisadas sobre argumentar y pensamiento argumentativo conformarían a esta competencia.

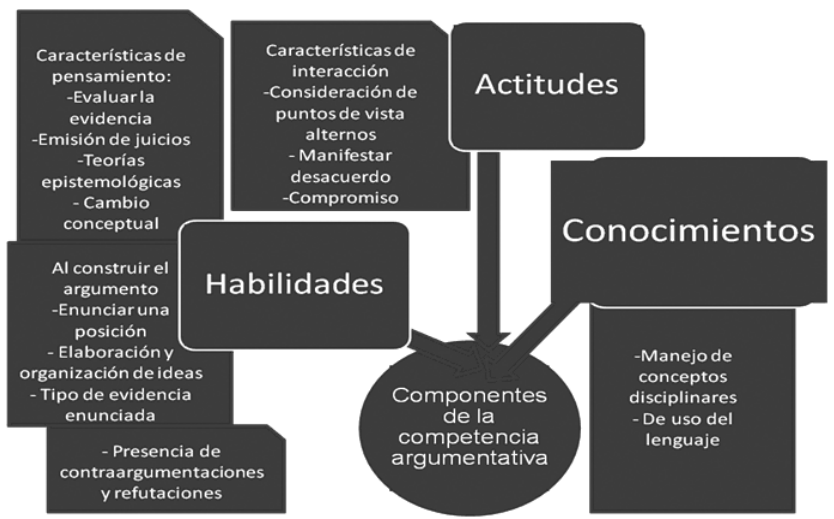

Figura 2. Recursos agrupados en componentes de la competencia argumentativa.

$\mathrm{Al}$ agrupar los recursos que constituyen la competencia argumentativa en los tres componentes de la noción de competencia propuestos desde el enfoque constructivista (véase Figura 2) se observa que el componente de habilidades (saber hacer) incluye varios recursos cognitivos, los cuales se articulan con los conocimientos (saber saber) y las actitudes (saber ser y convivir) para lograr un desempeño eficiente al ser parte de un argumento dialógico como el que debe brindar un foro de discusión en línea.

La construcción de un discurso convincente y coherente, apoyado en evidencia, como parte del pensamiento de orden superior, se da cuando los individuos tienen la posibilidad de poner en práctica sus habilidades (Kuhn, Goh, Iordanou y Shaenfield; 2008). El foro de discusión, dentro de procesos de formación en línea, se vuelve un espacio que permite la generación de razonamientos con base en aseveraciones y conocimientos científicos (Veerman, Andriessen y Kanselaar, 2000) al convertirse en un lugar donde se revisan posiciones frente a un hecho o situación con respecto a otras (Lewis, 2005) gracias al proceso de involucramiento en una discusión crítica para resolver diferencias de opinión así como productos simbólicos construidos o criticados durante un cuestionamiento (Nussbaum, Sinatra y Poliquin, 2008).

\section{Diseño educativo de los foros como situaciones de desarrollo de la competencia argumentativa}

Una competencia corresponde a situaciones que exigen el despliegue de sus componentes (Perrenoud, 2004). En este sentido, la perspectiva constructivista explica que las competencias no existen en abstracto, para poder describirla se necesita de una situación que permita, a su vez, reconocer los recursos que se movilizan, por lo que existen tantas competencias como familias de situaciones.

La cotidianidad presenta una serie de situaciones diferentes según la edad, la condición social y decisiones tomadas; para resolverlas, se recurre a la experiencia, la cual se traduce en la integración de innovación y repetición.

Por lo regular las competencias se construyen en situaciones que comparten similitudes que contribuyen a la progresión de la competencia. Por ejemplo, en los foros de discusión el estudiante puede desarrollar una actitud crítica y de pensamiento flexible a la vez que profundiza en un conocimiento disciplinar en la medida que el foro le ofrezca la oportunidad de manifestar desacuerdo al elaborar un argumento, contraargumento o refutación en un ambiente donde se promueve el respeto a la pluralidad de ideas y donde el objetivo sea deliberar alrededor de un tema importante en su profesión.

De allí que las competencias de un individuo dependan de las situaciones de práctica en que se encuentra a menudo, la pericia revela una forma de inteligencia situada al descubrir poco a poco las similitudes y analogías que no son evidentes. Desarrollar un enfoque situado para la competencia permite un abordaje desde las situaciones donde ocurren (Jonnaert, 2000, 2008).

En este sentido, se plantea que el foro de discusión es una experiencia situada, pues el debate es alrededor de temas con relevancia social relacionados con su formación, discusiones que no son particulares a los foros sino del ejercicio profesional de la disciplina.

En efecto, el desarrollo de las competencias se da en situaciones. En el caso de las instituciones educativas es necesario hablar de familias de situaciones para enriquecer esquemas de aprendizaje, lo cual llevaría directamente a una didáctica que promueva la interacción entre el docente y pares en prácticas socioculturales, el uso de un repertorio de procedimientos del estudiante con el objeto de que los individuos puedan enfrentar situaciones que les confronten y provoquen un desafío cuya solución demande recursos variados para dinamizar las interacciones, el diálogo y los afectos (Vergnaud, 2006).

En consonancia con el argumento anterior, Mason y Sirica (2006) señalan la necesidad de proveer a los estudiantes de diversas oportunidades de desarrollo de la argumentación (problemas desestructurados sin respuesta correcta) que permitan pensar y juzgar.

El debate generado en foros de discusión de formación universitaria, da oportunidad de construir una familia de situaciones que permitan un intercambio con objeto de expli- 
car una posición y que posibilita el despliegue de conocimientos, habilidades y actitudes, como escuchar, entender, responder, preguntar, interrumpir, aclarar, etcétera, y cuyo fin principal es que un estudiante aprenda a exponer sus pensamientos de manera coherente y articulada.

\section{Elementos a considerar en el diseño educativo de los foros}

Diferentes estudios (Mason y Sirica, 2006; Kanuka, Rourke y Laflamme, 2007; Yao, 2008; Lim, 2005; Garrison y Anderson, 2005) señalan aspectos a considerar al planear las situaciones de aprendizaje que pueden influir en el éxito o fracaso del debate que se promueve en los foros, entre esos factores se mencionan: la temática a discutir, el tiempo, la naturaleza del curso, la naturaleza del soporte en línea (plataforma), los recursos que ofrece, la presencia del líder y las instrucciones dadas. Brevemente analizaremos algunos de ellos.

El uso de temas controversiales puede ayudar a entender el conocimiento disciplinar (Mason y Sirica, 2006), es decir, proponen que las temáticas deben ser de importancia social para que las personas puedan comentar en principio sin necesidad de un conocimiento amplio y el fenómeno debe tener una estructura compleja e incierta. Estas características del tema permiten la construcción de un argumento dialógico que a su vez permite el uso de evidencia para soportar y probar las teorías a través de la construcción de contraargumentos y refutaciones.

Para que estos elementos se hagan presentes, se recomiendan preguntas que provoquen reflexiones (Kuhn, 1992) acerca de la certidumbre, puntos de vista alternativos y experiencias. Por ejemplo, ¿por qué estás tan seguro de esas causas?, ¿podría haber más de un punto de vista correcto acerca de esta temática?, ¿los expertos estan seguros acerca de las causas de esta situación?, ¿cómo sabes que es esa la causa?, ¿cuál es la razón de que algunas personas piensen de manera distinta a ti?, ¿qué podrían decir para mostrar que tú estás mal?, ¿los demás tendrían evidencia para mostrar que estás mal?

El diseño educativo de un foro de discusión debe incluir patrones de mensaje y respuestas, ya que mantienen organizada la conversación, cuidar el papel del tutor de manera que sea mayor en los primeros momentos y menor en los subsecuentes, así como apoyar la relación de los temas con las experiencias de los participantes (Kanuka, Rourke y Laflamme, 2007, Yao, 2008; Lim, 2005).

Un acuerdo de las investigaciones es que los métodos educativos basados en problemas como la webquest, el debate, la invitación a un experto, técnica de grupo nominal y deliberación reflexiva, son promotores de una mayor presencia cognitiva $^{3}$ (Kanuka et al. 2007).

\footnotetext{
${ }^{3}$ Categoría propuesta por Garrison y Anderson (2005) la cual es entendida como la reflexión continúa de los participantes alrededor del tema.
}

Las investigaciones (Coffin y Hewings, 2005; Coffin, 2004; Joiner y Jones, 2003; Hammond, 2000, 2005; De Smet, 2009; Ying, 2006) sobre el uso de foros aportan sustento asociados en al menos tres puntos para construir situaciones a partir de las cuales se puede apoyar al desarrollo de la competencia argumentativa conceptualizada como se ha venido exponiendo.

El primer punto es con respecto a la existencia de un desarrollo de pensamiento crítico y habilidades argumentativas bajo ciertas condiciones en el foro (existencia de un tutor, instrucciones claras, metas específicas, así se dan las características de un argumento).

El segundo aspecto es el desarrollo del pensamiento crítico en gran medida gracias al compromiso que se da en el argumento dialógico (producto del intercambio de ideas y entendimientos que surgen de discrepancias con el objetivo de llegar a un acuerdo) que se lleva a cabo.

El tercer punto hace referencia a los elementos de diseño educativo que influyen en la calidad del argumento como son la instrucción, el tipo de tarea y la atención diferenciada de las características de un buen argumento previo, dadas a los estudiantes, así como la temática puesta a discusión (un tema social que sea polémico brinda más posibilidades de participación).

Estos tres aspectos son resultado de situaciones educativas promotoras de un argumento dialógico en los foros de discusión de tal forma que los participantes muestran un compromiso de intercambio de ideas que permiten la construcción de conocimiento.

El reto es, entonces, ofrecer situaciones sólidas y fecundas que giren alrededor de saberes importantes que proporcionen desafíos en la formación para desarrollar competencias genéricas y competencias argumentativas en particular.

\section{Conclusiones}

Desde la visión que ofrece el enfoque de competencias, se observa que una competencia importante por promover es la argumentativa. Esta importancia radica en que, a partir de ella, los estudiantes en formación aprenden a fundamentar sus puntos de vista o a refutar ideas que les parecen incorrectas o incompatibles con su pensamiento, como producto del beneficio directo que les da el entendimiento de un lenguaje científico.

En la Educación Superior, se asume que los estudiantes deben desarrollar la capacidad de presentar argumentos bien fundamentados, capacidad que debe acompañarse de una actitud crítica hacia el conocimiento con el fin de ser parte de sus herramientas para el desarrollo del conocimiento profesional. Pese a ello, las investigaciones muestran que la argumentación de los adultos con estudios en este nivel es limitada e insuficiente (Joiner y Jones, 2003; Kuhn y Udell, 2003).

Las situaciones profesionales y científicas exigen una capacidad de argumentar, compartida con eventos de la vida cotidiana, por lo que un individuo debe poder explicarse, ob- 
tener información, justificar un comportamiento, hacer comprensibles sus elecciones, defender sus derechos y su autonomía. De allí que la argumentación deba ser reconocida como pieza central en el discurso de las disciplinas y no sólo como una parte de las habilidades comunicativas, de acuerdo con Kuhn en que argumentar es el proceso por el cual el pensamiento es manifestado y que el pensamiento crítico surge en discusión (argumento dialógico).

Las instituciones educativas en estos momentos se encuentran interesadas en procesos de formación en línea, el interés se debe, en gran parte, a que está disponible en cualquier lugar en que estén ubicados los alumnos y se acomoda a los tiempos del estudiante, es decir, ofrece alternativas de ritmo, metodología y formatos; aunado a que en un sentido amplio, buscan desarrollar capacidades en el estudiante que le permitan enfrentar problemas de forma flexible, al mismo tiempo que se le anima a forjar una autonomía en cuanto a la evolución de sus aprendizajes y el conocimiento de sí mismo como aprendiz, permitiéndole el conocimiento de sus posibilidades de autoformación (Basabé, 2007; UNESCO, 2008; Mauri y Onrubia, 2008).

$\mathrm{Al}$ respecto, se vuelve necesario entender los procesos de aprendizaje y enseñanza que tienen como escenario la modalidad específica de la educación en línea en la formación de profesionales, es decir, se debe comprender cómo se desarrollan estos procesos en los estudiantes para incorporar en los programas situaciones educativas que los fortalezcan. En ese sentido, se considera a la argumentación como una capacidad necesaria en esos procesos de formación, debido a que es un contexto en el cual no sólo es deseable sino que será una de las formas en la cual los estudiantes demuestran sus aprendizajes.

Entre las ventajas encontradas en el uso del foro de discusión es que el debate es importante para un cambio conceptual ya que ofrece puentes entre la experiencia personal, las circunstancias y los conocimientos. Debido a que la construcción de un argumento implica tomar decisiones en la

\section{Referencias}

Amestoy, M. (2002). La investigación sobre el desarrollo y la enseñanza de las habilidades de pensamiento. Revista Electrónica de Investigación Educativa, 4(1), 1-32. Recuperado de http://redie.uabc.mx/vol4no1/contenidoamestoy.html

Basabé, P. (2007). Educación a distancia en el nivel superior. México D. F., México: Trillas.

Clarck, D. B., Stegmann, K., Weinberger, A., Muhsin, M. y Erkens, G. (2008). Technology-enhanced learning environments to support students argumentation. En S. Erduran y M. P. Jiménez-Aleixandre (Eds.), Argumentation in science education: perspectives from classroom-based research (pp. 91-115). Dordrecht, The Netherlands: Springer.

Coffin, C. (2004). Arguing about how the world is or how the world should be: the role of argument in IELTS tests. Journal of English Science Education, 3 , 229-246.

Coffin, C. y Hewings, A. (2005). Engaging electronically: using CMC to develop students argumentation skills in higher education. Language and education, 19 (1), 32-49.

Coffin, C., North, S. y Martin, D. (2009). Exchanging and countering points of view: a lingüístic perspective on school students' use of electronic conferencing. Journal of Computer Assisted Learning, 25, 85-98. adaptación del contenido, al momento de la conversación y al destinatario, se hablaría de una competencia que refleja el poder sostener un punto de vista sin huir a la confrontación, al mismo tiempo que se escucha a los otros para conocer sus opiniones, es decir, se pondrían en movimiento una serie de recursos, frente a una situación compleja por resolver (Perrenoud, 1999).

Aquí se explora una aproximación a la argumentación diferente a las que se habían planteado anteriormente, donde se entiende son varios los recursos que el individuo pone en marcha en una acción situada. La concepción de competencia argumentativa obedece a una integración de conocimientos, habilidades y actitudes al interactuar en una argumentación dialógica presente en un foro de discusión cuyo objetivo es promover la deliberación alrededor de un tema.

Bajo el enfoque cognitivo-constructivista y el escenario de foro de discusión en línea la competencia argumentativa como producto de esta revisión de la literatura se entiende como la integración de conocimientos, actitudes y habilidades las cuales se movilizan en la defensa o rechazo de una aseveración, hecho o conclusión a través de una exposición coherente, fundamentada y sistemática, cuyo objetivo es plantear postura a la vez que se comparten significados y se negocia un consenso.

En síntesis, se propone estudiar a las argumentaciones como una competencia en ejecución en los foros de discusión debido a la función social en la comunicación que han adquirido en la interacción virtual, cuando son asumidos como un elemento importante en la formación a distancia de última generación (Basabé, 2007), se les retoma cada vez más en la educación formal universitaria y a partir de la investigación documental realizada se observan lagunas sobre la explicación del desarrollo de la cognición de los individuos en entornos virtuales de aprendizaje (es una línea de investigación en desarrollo) y además son un espacio fértil en la promoción de la capacidad para argumentar.

Coll, C. (2007). Las competencias en educación escolar: algo más que una moda y mucho menos que un remedio. Aula de Innovación Educativa, 161, 34-39.

Coll, C. (2009). Los enfoques educativos basados en competencias y el sentido de aprendizaje escolar. Conferencia magistral presentada en el X Consejo Mexicano de Investigación Educativa. Recuperado de http://www.youtube.com/watch?v=12xaCoo_09I

Delgado, G. A., Borge, B., García, A. J., Oliver, C. R., y Salomon, S. L. (2005). Competencias y diseño de la evaluación continua y final en el EEES. Programa de Estudios y Análisis de Ministerio de Educación y Ciencia. Catalunya. Recuperado de http://nevada.ual.es:81 /ufid/archivos/competencias\%20evaluacion\%20ees-MEC.pdf

De Smet, M., Van Keer, H. y Valcke, M. (2009). Cross-age peer tutors in asynchronous discussion groups: a study of the evolution in tutor support. Instructional Science, 37, 87-105.

De Zubiría, J. S. (2006). Las competencias argumentativas encontrando la visión desde la educación. Bogotá, Colombia: Aula Abierta.

Delamare, L. D. F. y Wingerton, J. (2005). What Is Competence? Human Resource Development International, 8(1), 27-46.

Fu-Ren, L., Lu-Shih, H. y Fu-Tai, C. (2009). Discovering genres of online discussion threads via text mining. Computers \& Education journal, 52, 481-495. 
Garrison, D. R. y Anderson, T. (2005). El e-learning en el siglo XXI: investigación y práctica. Madrid, España: Octaedro.

Golanics, D. D. (2008). Enhancing online collaborative argumentation through question elaboration and goal instructions. Journal of Computer Assited Learning, 24, 167-180.

González, J., Wagenaar, R. y Beneitone, P. (2004). Tuning-América Latina: un proyecto de las universidades. Revista Iberoamericana de Educación, 35, 151-164. Recuperado el 27 de octubre de 2009, de http:/ / www.rieoei.org/rie35a08.htm

Gútierrez, L. A. E. (2004). La comunicación didáctica en los espacios electrónicos de aprendizaje en la educación a distancia. Tesis de doctorado no publicada. Facultad de Ciencias Políticas y Sociales, Universidad Nacional Autónoma de México, México D.F., México.

Guzmán-Cedillo, Y. I. (2010). Une grille d'évaluation pour déterminer le niveau de la compétence argumentative. Recuperado http://www.matimtl.ca/journeesMati/journee2011/index.jsp

Hammond, M. (2000). Communication within on-line forums: the opportunities the constraints and the value of communicative approach. Computer and Education, 35, 251-262.

Hammond, M. y Wiriyapinit, M. (2005) Learning through online discussion: A case of triangulation in research. Australasian Journal of Educational technology, 21(3), 283-302.

Häkkinen, P. y Järvela, S. (2004). Sharing and constructing perspectives in webbased Conferencing. Computers \& Education, 47, 433-447.

Joiner, R. y Jones, S. (2003). The effects of communication medium on argumentation and the development of critical thinking. International Journal of educational research, 39, 861-871.

Jonnaert, P. (2000). La thèse socioconstructiviste dans les nouveaux programmes d'études au Québec: un trompe l'oeil épistémologique? Canadian Journal of Science, Mathematics and Technology Education, 1(2), 223-230.

Jonnaert, P., Barrette, J., Masciotra, D., y Yaya, N. (2008). La competencia como organizadora de los programas de formación: hacia un desempeño competente. Profesorado. Revista de currículum y formación del profesorado, 12(3), 1-32.

Kanuka, H., Rourke, L. y Laflamme, E. (2007). The influence of instructional methods on the quality of online discussion. British Journal of Educational Technology, 38(2) 260-271

Kuhn D., Goh, W., Iordanou, K. y Shaenfiel, D. (2008). Arguing on the computer: a microgenetic study of developing arguments skillls in a computersupported environment. Child Development, 79(5), 1310-1328.

Kuhn, D., Iordanou, K., Pease, M. y Wirkala, C. (2008). Beyond control of variables: what needs to develop to achieve skilled scientific thinking? Cognitive Development, 23, 435-451.

Kuhn, D. y Park, S. H. (2005). Epistemological understanding and the development of intellectual values. International Journal of Educational Research, 43, 111 124.

Kuhn, D. y Udell, W. (2003). The development of argument skills. Child Development, 74(5), 1245-1260.

Kuhn, D., Cheney, R. y Weinstock, M. (2000). The developmental of epsitemological understanding. Cognitive Development, 15, 309-328.

Kuhn, D. y Lao, J. (1998). Contemplation and conceptual change: integrating perspectives from social and cognitive psychology. Developmental Review, 18 , $125-154$

Kuhn, D. (1992). Thinking as argument. Harvard Educational Review, 62(2), 155175.

Lewis, D. (2005). Arguing in English and French asynchronus online discussion. Journal of pragmatics, 37, 1801-1818.

Lim, C. (2005). The use of online forums to support inquiry in a PBL environment: observations from a work-in-progress. British Journal of Educational Technology, 36(5), 919-921.

Marttunen, M. (1997). Electronic mail as a pedagogical delivery. Research in Higher Education, 38(3), 345-362

Marttunen, M. (1997). Teaching argumentation skills in an electronic mail environment. Innovations in Educational and Teaching International, 34(3), 208218.

Mason, L. y Scirica, F. (2006). Prediction of students' argumentation skills about controversial topics by epistemological understanding. Learning and Instruction, 16, 492-509.

Mauri, T. y Onrubia, J. (2008). Dimensiones e indicadores de la calidad de los procesos formativos en línea: pautas para el análisis. En E. Barberá, T.
Mauri, J. Onrubia, G. Aguado, A. Badia y C. (Eds.), Cómo valorar la calidad de la enseñanza basada en las TIC. Pautas e instrumentos de análisis (pp. 99-119). Barcelona, España: Graó.

Mazzolini, M. y Maddison, S. (2003). Sage, guide or ghost? The effect of instructor intervention on student participation in online discussion forums. Computer \& Education, 40, 237-253.

Mulder, M., Weigel, T. y Collings, K. (2008). El concepto de competencia en el desarrollo de la educación y formación profesional en algunos estados miembros de la UE: un análisis crítico. Profesorado. Revista de Curriculum y For mación del Profesorado, 12(3), 1-23.

Mulder, M., y Collins, K. (2007). Competence Development in Public and Private Or ganizations: a Survey of Its Use in Practice in Selected EU Member State. Comunicación presentada en la ECER Conference, Gent (Belgium).

Nussbaum, E. M., Sinatra G. M. y Poliquin, A. (2008). Role of epsitemic beliefs and scientific argumentation in science learning. International Journal of Science Education, 30 (15), 1977-1999.

Peon, Z. M. (2004). Habilidades aroumentativas de alumnos de primaria y su fortalecimiento (Tesis de doctorado no publicada). Facultad de Psicología, Universidad Nacional Autónoma de México, México DF, México.

Perrenoud, P. (2008). Construir competencias desde la escuela. Santiago, Chile: J. C. Sáez.

Perrenoud, P. (2009). Dię nuevas competencias para enseñar. 7a ed. México: GRAO.

Perrenoud, P. (2004). Introducción: nuevas competencias profesionales par enseñar. En P. Perrenoud (Eds.), Diez nuevas competencias para enseñar. invitación al viaje (pp. 1-16). Barcelona, España: Graó.

Perrenoud, P. (1999). L'école saisie par les competences. Recuperado de http://www.unige.ch/fapse/SSE/teachers/perrenoud/php_main/php_199 9/1999_14.html

Posada, A. R. (1999). Formación superior basada en competencias interdisciplinariedad y trabajo autónomo del estudiante. Recuperado de http://www.rieoei.org/deloslectores/648Posada.pdf

Rodríguez, B. L. I. (2004). El modelo argumentativo de Toulmin en la escritura de de artículos de investigación educativa. Revista digital universitaria, 5(1). Recuperado de http://www.revista.unam.mx/vol.5/num1/art2/art2.htm

Shön, D. (1998). El profesional reflexivo: como piensan los profesionales cuando actúan. Barcelona, España: Paidós.

Sampson, V. y Clarck, D. (2008). Assesment of the ways students generate arguments in science education: current perspectives and recommendations for future directions. Science Education, 92(3), 447-472.

Tardif, J. (2006). L'évaluation des compétences: documenter le parcours de développement. Montréal: Chenelière-éducation.

Tobón, S., Rial, A., Carretero, M. y García, J. A. (2006). Competencias, calidad educación superior. Bogotá, Colombia: Alma Mater Magisterio.

UNESCO (2008). Estándares de competencias en TIC para docentes. Marco de políticas educativas. Recuperado http://www.eduteka.org/EstandaresDocentesUnesco.php

Van Eemeren, F. H., Houtlosser, P. y Snoeck, F. (2008). Dialectical profiles and indicators of argumentative moves. Journal of Pragmatics, 40, 475-493.

Van Eemeren, F. H. y Houtlosser, P. (2007). The study of argumentation as normative pragmatics. Pragmatics of Cognition, 15(1), 161-177.

Van Eemeren, F. H. y Grootendorst, R. (1992). Argumentation, comunication and fallacies: A pragma-dialectical perspective. New Jersey, USA: Lawrence Erlbaum.

Veerman, A. L., Andriessen, J. E. y Kanselaar, G. (2000). Learning through syncronus electronic discussion. Computer \& Education, 34, 269-290.

Vergnaud, G. (2006). Les competences, bravo ! mais encore ? Réflexions critiques pour avancer. Recuperado de http://www.pedagopsy.eu/competences_vergnaud.htm

Weinert, F. E. (2001). Concept of competence: a conceptual clarification. En D. S. Rychen y L. H. Salganik (Eds.), Defining and selecting key competencies. Göttingen, Alemania: Hogrefe.

Weigand, E. (2006). Argumentation the mixed game. Argumentation, 20(1), 59-87.

Willard, C. (1983). Argumentation and the social grounds of knowledge. Alabama, USA: University of Alabama Press.

Ying-Hua, G., Chin-Chung, T. y Fu-Kwun, H. (2006). Content analysis of online discussion on a senior-high-school discussion forum of a virtual physics laboratory. Instructional Science, 34, 279-311.

(Articulo recibido: 28-6-2010; revisado: 6-1-2013; aceptado: 13-1-2013) 